\title{
Forecasting mortality: when academia meets practice
}

\author{
Séverine Gaille
}

Received: 2 August 2011/Revised: 15 November 2011/Accepted: 22 November 2011 /

Published online: 7 February 2012

(C) DAV / DGVFM 2012

\begin{abstract}
Techniques used in practice often differ from tools developed in academia. The lack of communication that may exist between academia and practice can then have important consequences for many insurance companies or pension funds. This issue is illustrated with what is currently happening in Switzerland. Swiss pension funds use mortality tables that are regularly updated with new observations. A new version of these tables has been recently published and includes a procedure to forecast mortality until 2150. The method applied for these projections is very different from the several forecasting models that have been developed in academia over the last decades. In this paper, we compare mortality forecasts used by practitioners in Switzerland and the forecasts resulting from two simple approaches well-known in academia, the Lee-Carter model and the Heligman-Pollard function. These two approaches have the advantage of simplicity and thus, all insurance companies and pension funds may implement them without any difficulties. The analysis demonstrates that both academic methods forecast a more important decrease in mortality than the approach applied by pension funds, especially in the long-run and for females. Impacts on pension liabilities are then evaluated, enlightening the future challenges many institutions will face. Finally, a few points which insurance companies or pension funds need to be cautious with, when using mortality forecasts, are summarized.
\end{abstract}

Keywords Mortality forecasts · Pension fund liabilities - Switzerland - LPP 2010 . Lee-Carter model · Heligman-Pollard function

S. Gaille (ه)

Department of Actuarial Science, Faculty of Business and Economics,

University of Lausanne, 1015 Lausanne, Switzerland

e-mail: Severine.Gaille@unil.ch 


\section{Introduction}

Longevity risk became an important and highly discussed topic in nowadays developed economies. When analyzed, this risk is often divided in two components, an individual one and an aggregate one. The first one refers to random fluctuations around an expected value and is due to the uncertainty in an individual's lifetime. Insurance companies reduce this risk by insuring a sufficiently large number of persons. The second component is a systematic risk, that is a systematic deviation from the expected lifetime due to unexpected mortality improvements. In such a situation, individuals tend to constantly outlive their savings. Such mortality improvements have significant financial impacts on insurance companies as well as on the social security system of a country, on its health care system, and on many other institutions including pension funds and banks. Indeed, an increasing life expectancy is reflected in the potential costs of financing a growing proportion of older aged retired individuals.

Mortality modeling has then attracted increased research attention over the last decades in several disciplines. As life expectancy, and its associated costs to society, has increased significantly, actuaries started to be interested in such models. Demographers look for explanations of past evolutions. Biologists are interested in estimates of the limit to human life span, that is the biologically maximum length of life, and look to genetics as an explanation of the increase in life expectancy. Economists analyze the impact of life expectancy on economic growth. Many experts are also interested in knowing if the increase in life expectancy is due to longer medicine intake or to improved health. Answers to such questions are key factors to determine the costs of healthcare for the elderly. Several models were then developed in academia in order to capture the features of mortality rates (see Booth and Tickle [5] for a review).

However, in Switzerland, none of these techniques is used in practice. Indeed, actuarial valuations for pension funds are mainly based on three different official mortality tables. The first one refers to EVK ("Eidgenössische Versicherungskasse"), based on the experience of the Federal Pension Fund, and is published every 10 years since 1950 . The second one, published every 10 years from 1950 to 2000 and every 5 years since then, is called VZ ("Versicherungskasse der Stadt Zürich") and is mainly based on the experience of the pension fund of the City of Zurich and, since 2005, also on the experience of several other public pension funds. Finally, in 2002 , the first mortality tables based on the experience of private pension funds were published, denoted BVG 2000 ("Bundesgesetz über die berufliche Alters-, Hinterlassenen- und Invalidenvorsorge") or LPP 2000 ("loi sur la prévoyance professionnelle vieillesse, survivants et invalidité"). These tables are updated every 5 years.

The current tables are then first VZ $2005^{1}$, based on the statistics of the years 2001-2005, second EVK 2000, based on 1993-1998 and no longer updated, and third LPP 2010 based on the observations of 2005-2009. VZ 2005 and LPP 2010 are different from previous tables since they include a forecasting approach for

\footnotetext{
1 A new version of these tables is expected for November 2011.
} 
mortality rates until 2150. Both forecasting approaches are based on a work published by the statistical office of the canton Vaud (Menthonnex [16], updated a few years later with Menthonnex [17]). The forecasts do not use any models developed in academia, but mainly expert opinions or "informed judgement" on potential future evolutions as well as "historical continuity"2. Although this approach does not rely on well-known techniques, it gives an interesting and different perspective on the possible future trends.

One may wonder then what would be the financial impact for a pension fund if techniques developed in academia were actually employed in practice. This paper tries to answer this question by applying two different forecasting approaches to Swiss mortality rates and comparing the financial impacts they have. Indeed, future mortality rates are key components in assessing retirement liabilities within pension fund valuations.

The first approach refers to the well-known Lee-Carter model (Lee and Carter [12]), which has been successfully applied at the population level for the US data in the past. This model has become a standard in mortality projections. It allows trends to vary by age, while a single common factor across ages is used in order to determine the general level of mortality improvement over time. Its popularity is mainly related to its simplicity of use as a single time series needs to be projected in order to forecast the complete age profile of mortality.

We suggest as well a second approach. A decade before the Lee-Carter model, Heligman and Pollard [10] developed a mathematical expression for the graduation of the age pattern of mortality. In contrast to the Lee-Carter model designed for projections, their function is aimed at smoothing the age pattern of mortality, each parameter having convenient demographic meaning. However, over the years, several applications and extensions of the Heligman-Pollard model have been suggested, including attempts to use this model as a tool to forecast mortality rates over time (Bell [1], Felipe et al. [8], McNown and Rogers [14], Rogers and Gard [20]). The principal benefit of the Heligman-Pollard function over the Lee-Carter model is that it does take advantage of the strong constancy observed in the age profile of mortality rates. Furthermore, the use of a parametric model enables comparisons over time and across countries.

Ideally, the time series of the parameters should be modeled with multivariate time series, which capture dependence between the parameters. However, univariate AutoRegressive Integrated Moving Average (ARIMA) models have been commonly used in the past, as univariate forecasts are more easily understood and applied. The approach introduced in this paper also uses univariate models, but not traditional ARIMA processes. Indeed, the suggested method is even simpler as it uses standard regression methods. Experts using such an approach may include in their forecasts some prior-knowledge which accounts for the impact on mortality of known factors. Several assumptions on the future evolutions may then be tested. ARIMA processes can not anticipate a decline in mortality due to a new cure against, for instance, cancer, in contrast to the approach presented in this paper. Besides, including expert knowledge partly allows to include some dependences

\footnotetext{
$\overline{2}$ Terminology used by McNown and Rogers [14].
} 
among the parameters in the model, as some implausible forecasted trends for the parameters may be adjusted to the expert expectations, based on past observations.

These two forecasting methods are chosen because of their simplicity. They can be easily implemented by practitioners as they do not require high qualifications in specific fields, such as multivariate time series. These two models are presented in the third section of the paper after an introduction to the dataset in Sect. 2. In Sect. 4, the two models are tested on Swiss data. Based on the fitting period of 1876-1979, female mortality projections are performed until 2005, which enables comparisons with actual data and thus, to measure the accuracy of the forecasts. Following the same procedure as in the first part of the paper, mortality is then projected over the period 2006-2075 in Sect. 5, giving key results on possible future trends. A comparison with the forecasting approach of the LPP 2010 tables is performed. Section 6 enlightens the impact of the mortality assumptions on the valuation of pension liabilities through the pension conversion rate. The last section draws some conclusions and highlights some cautions to be taken when forecasting mortality.

\section{Data}

Mortality rates are defined as the number of persons for each age and sex who die in a particular year, divided by the number of persons of that age and sex alive at the beginning of the year. Such data are collected from the Human Mortality Database, administered by the Department of Demography of the University of California and by the Max Planck Institute for Demographic Research (Berkeley, USA and Rostock, Germany [2]). This database contains demographic information by single age (zero to 110) for years 1876-2005. Several countries are represented in it, including Switzerland for which the main data source is the Swiss Federal Statistical Office. Mortality tables introduced in this paper are based on the age-last-birthday rule.

\section{Models}

\subsection{Heligman-Pollard model}

In 1980, Larry Heligman and John H. Pollard presented a new function to model the age pattern of mortality based on the Thiel's formulation (Thiel [23]). Their function is a concise representation of mortality by age, since the most important features of the changing age pattern of mortality are captured by a relative small set of parameters. Several versions of the Heligman-Pollard function exist. Two of them are described in some details thereafter, one with eight parameters, and the other one with nine.

The Heligman and Pollard [10] function is defined as ${ }^{3}$

\footnotetext{
3 In order to avoid to overload the equations, the sex index is omitted.
} 


$$
\frac{q_{x, t}}{p_{x, t}}=A_{t}^{\left(x+B_{t}\right)^{C_{t}}}+D_{t} e^{-E_{t}\left(\ln (x)-\ln \left(F_{t}\right)\right)^{2}}+G_{t} H_{t}^{x}
$$

where

$$
\begin{aligned}
q_{x, t}= & \text { probability of dying at age } x, \text { in year } t ; \\
p_{x, t}= & 1-q_{x, t} ; \\
Z_{t}= & \text { value of the parameter } Z \text { at time } t \\
& \text { for } Z \in(A, B, C, D, E, F, G, H) .
\end{aligned}
$$

An interesting feature of this function is that each parameter has a demographic and/or biological meaning. The function is a sum of three terms: the first one represents the mortality rates during childhood; the second one, the middle ages (accident hump); and the last one, the mortality rates at older ages. This structure allows the inclusion in the model of several assumptions about the effects that changes in behavioral or socioeconomic variables may have (Tabeau et al. [22]).

The mortality rates during childhood are expressed as a function of three parameters: Parameter $A$ is the level of infant mortality, $B$ describes the mortality change between age zero and age one (the larger the value of $B$ is, the smaller the change) and $C$ defines the speed at which the mortality rates decline at young ages.

The accident hump is modeled by three parameters, as well. Parameter $D$ describes the severity of the accident hump (the larger the value is, the higher the hump). Parameter $E$ specifies the age range at which the accident hump occurs, while $F$ is the age for which the hump has the most impact.

Finally, the mortality at older ages is modeled by a Gompertz function. The general level of mortality rates is reflected by the parameter $G$, and $H$ is needed to characterize the steepness of the curve.

Heligman and Pollard suggested as well some alternative representations of the age profile of mortality. One of them is found by noting that Eq. 1 is almost identical to

$$
q_{x, t}=A_{t}^{\left(x+B_{t}\right)^{C_{t}}}+D_{t} e^{-E_{t}\left(\ln (x)-\ln \left(F_{t}\right)\right)^{2}}+\frac{G_{t} H_{t}^{x}}{1+G_{t} H_{t}^{x}} .
$$

Indeed, $\frac{q_{x, t}}{p_{x, t}} \approx q_{x, t}$ for young ages as $p_{x, t} \approx 1$. Equation 2 is only adjusted in its third part, the one reflecting the mortality rates at older ages. This leads to an alternative formulation of the form

$$
q_{x, t}=A_{t}^{\left(x+B_{t}\right)^{C_{t}}}+D_{t} e^{-E_{t}\left(\ln (x)-\ln \left(F_{t}\right)\right)^{2}}+\frac{G_{t} H_{t}^{x}}{1+K_{t} G_{t} H_{t}^{x}} .
$$

An additional parameter $K$ is added in order to have a better fit at older ages. It allows the age pattern of mortality (the curve representing the logarithm of mortality rates) to be concave upward, contrarily to Eq. 1. The difference is important here: A concave downward curve means that the logarithm of mortality rates at older ages increases, but at slower rates as age increases. It would indicate that the age pattern of mortality has a global maximum. In contrast, a concave upward curve implies 
log-mortality rates growing at a faster pace as age increases. Depending on the data, including parameter $K$ in the Heligman-Pollard function may then be useful.

\subsubsection{Estimation}

The parameters of the Heligman-Pollard function are estimated by a weighted least squares, the weights used implying a minimization of the relative error as suggested by Heligman and Pollard [10]. The maximal age included in the model is increased from 94 to 101 in 1970 as data for older ages become more reliable. The following constraints are set on the parameters of the function:

- $A, B, C, D, G \in(0,1)$;

- $E, H>0$;

- $15 \leq F \leq 100$.

This weighted least squares results in a non-linear minimization. Several algorithms exist to solve non-linear equations and many languages and environments for statistical computing contain such functionality. A non-linear minimization requires initial estimations for the parameters. Starting values need to be as precise as possible for the algorithm to converge to an optimum, which may lead to some difficulties. By plotting the Heligman-Pollard function on the same graph with the actual mortality, one can read its initial values.

\subsubsection{Forecasts}

The estimation of the Heligman-Pollard function yields a set of observations on each parameter over time. Univariate models can then be applied to the fitted values of the parameters in order to forecast their temporal evolution and thus to project the complete age profile of mortality. Three functions are considered to model the trend of these parameters, that is

- Linear: $a \cdot t+b$;

- Exponential: $b \cdot \exp [a \cdot(t-1875)]$;

- Quadratic: $a \cdot t^{2}+b \cdot t+c$;

where $t$ represents the considered year. These functions have a simple structure, few parameters and give plausible forecasts, which are the required specifications in this study.

Each of these functions is fitted by ordinary least squares. The retained functions are the ones with the lowest Bayesian Information Criterion (BIC) for which all coefficients are significantly different from zero at a one percent significance level. However, in order to have sensible results, specific assumptions on the future trend of the coefficients of the Heligman-Pollard function are also considered. Indeed, the practical meaning of the parameters gives us clues on what might happen in the future and, then, should be taken into consideration in the projections. 
Plots of the parameter history may show a marked change of pattern during the beginning of the twentieth century, which may pose major problems in forecasting the parameters. We experimented several models, both working on the complete set of observations (1876-1979 or 1876-2005) as well as on smaller sets. We present in the following analysis the most relevant model according to the forecasting performance. Indeed, models predicting unreasonably high or low level of mortality are discarded. Only the most credible functions and most appropriate periods are used to forecast the value of the parameters.

\subsection{Lee-Carter model}

The initial model of Lee and Carter [12] decomposes the logarithm of the central death rates in two components: one describing the age pattern of average mortality rates; the other for a common time trend with differential impacts by age. In 2000, Lee suggested to model the force of mortality instead of the central death rate so that the implied death rates will be between zero and unity (Lee [11]). The model is then defined as

$$
\ln \mu_{x, t}=\alpha_{x}+\beta_{x} \kappa_{t}+\epsilon_{x, t}
$$

where

$\mu_{x, t}=$ force of mortality at age $x$, in year $t$;

$\alpha_{x}=$ mean value over time, at age $x$, of the logarithm of

the force of mortality;

$\beta_{x}=$ relative speed of mortality change at age $x$.

It reflects the impact of the time trend represented by $\kappa_{t}$

on age, that is the higher its absolute value, the greater the impact

of mortality changes over time on age $x$;

$\kappa_{t}=$ mortality rate trend over time;

$\epsilon_{x, t}=$ historical influences not captured by the model;

$=$ errors with mean zero and variance $\sigma^{2}$ (homoscedasticity).

To allow this interpretation of the parameter $\alpha_{x}$, the sum over the estimator $\hat{\kappa}_{t}$ is set equal to zero,

$$
\sum_{t=t_{\min }}^{t_{\max }} \hat{\kappa}_{t}=0 .
$$

Indeed, if $N$ is the number of years under observation,

$$
\begin{aligned}
\prod_{t=t_{\min }}^{t_{\max }} \mu_{x, t} & =\exp \left(\hat{\alpha}_{x} N+\hat{\beta}_{x} \sum_{t=t_{\min }}^{t_{\max }} \hat{\kappa}_{t}\right) \\
& =\exp \left(\hat{\alpha}_{x} N\right)
\end{aligned}
$$


which leads to

$$
\hat{\alpha}_{x}=\frac{1}{N} \sum_{t=t_{\min }}^{t_{\max }} \ln \mu_{x, t}, \quad \forall x .
$$

In order to have an identifiable model, another constraint on the parameters is specified, which usually is

$$
\sum_{x=x_{\min }}^{x_{\max }} \hat{\beta}_{x}=1 .
$$

The Heligman-Pollard function refers to mortality rates- $q_{x, t}$-whereas the LeeCarter model focuses on the force of mortality. Age-specific mortality rates are assumed to be constant within bands of age and time so that the force of mortality is determined using

$$
q_{x, t}=1-p_{x, t}=1-\exp \left(-\mu_{x, t}\right)
$$

where

$$
\mu_{(x+\delta),(t+\tau)}=\mu_{x, t}, \quad 0 \leq \delta, \tau<1
$$

\subsubsection{Estimation}

The parameters are estimated numerically using maximum likelihood estimation, assuming that the number of deaths at age $x$ follows a Poisson distribution with mean $l_{x, t} \cdot \mu_{x, t}, l_{x, t}$ being the population of age $x$ alive at the beginning of year $t$ (for a complete and clear description of this approach, see Delwarde and Denuit [7], pp 219-224). Several environments for statistical computing contain functions fitting the Lee-Carter model on some datasets (see, for example, the demography package for $R$ of Hyndman, function $l c a$ ).

\subsubsection{Forecasts}

A single common factor across ages is used for determining the general level of mortality improvement over time, and thus, a single time series $\left(\kappa_{t}\right)$ needs to be projected in order to forecast the complete age profile of mortality. An ARIMA process is fitted to the time series of $\kappa_{t}$, as suggested by Lee and Carter. The appropriate ARIMA process is determined through the procedure described by Pandit and $\mathrm{Wu}$ [19]. According to the forecaster's preferences, the approach introduced in Sect. 3.1.2 could be used instead.

The Heligman-Pollard model is fitted on smaller sets of observations than the complete one, as described in Sect. 3.1.2. The same procedure needs to be applied to model the time series $\kappa_{t}$. Indeed, as mentioned by Lee and Miller [13], assuming that the relative speed of mortality decline does not change over time, that is parameter $\beta_{x}$ is fixed over time, may produce non-realistic forecasts. Such an assumption does not apply for extended periods as 1876-2005. The simple solution 
proposed by Lee and Miller [13] is to reduce the period on which the model is fitted to 1950-2005, which is the approach followed in this paper.

\section{Test on Swiss data}

Before any forecasting attempts, one should verify that the Heligman-Pollard and Lee-Carter models are good representations of Swiss mortality. Thus, the two models are fitted to female mortality over the period 1876-1979 (and some smaller sets) to produce forecasts through 2005, as described in Sect. 3. The obtained estimates are then compared with the actual mortality rates over the same period, 1980-2005.

\subsection{Heligman-Pollard model}

\subsubsection{Step 1: fit}

The Heligman-Pollard function is fitted to the mortality rates over the period 1876-1979 (Fig. 1). The estimates for the parameters obtained through the constrained minimization process are not all within the defined limits. Between 1949 en 1979, the accident-hump parameter $F$ reaches the upper bound of 100 seven

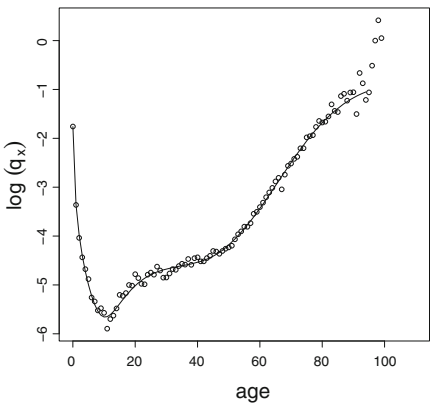

(a)

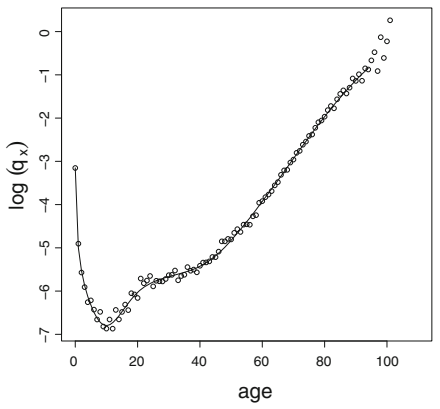

(b)

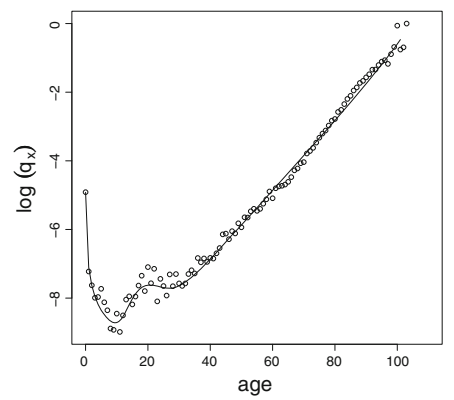

(c)

Fig. 1 Sample years of actual log-mortality rates (dots) and fitted Heligman-Pollard model (curve), females: a 1885, b 1935, c 1979 
times. Hence, the accident-hump parameters $D, E, F$ and the mortality-for-olderages parameter $G$ are non significantly different from zero even at a ten percent significance level. The same is true for the child-mortality parameters $A$ and $B$ for the one year when $B$ reaches the value of one. This issue is well-known and is due to multicollinearity. Indeed, this multicollinearity causes irregular changes of the parameter estimates from one year to another even if the mortality rates of adjoining years are similar (Bell [1], Tabeau et al. [21]). The solution usually adopted in the past is to fix the value of some parameters prior to the fitting process (Gaille and Sherris [9], McNown and Rogers [15], Tabeau et al. [21]). However, since such an approach yields to some loss in the fitting precision and for simplification purpose, we prefer to discard these years from further analysis.

\subsubsection{Step 2: projection of the parameters}

Univariate models are then applied to the fitted values of the parameters, so that their future trend may be estimated, resulting in:

Parameter A: As this parameter reflects the level of infant mortality, it is reasonable to observe a substantial decrease at the beginning of the twentieth century (hygiene improvement, vaccination discovery, etc). The exponential form is fitted by ordinary least squares over the period 1910-1979 and appears to be the most appropriate one (Fig. 2a; Tables 1, 2).

Parameter $B$ : The linear and exponential decreases have the same BIC and in both cases coefficients are significant at a one percent significance level. However, future infant mortality rates resulting from such a decrease would be absurdly high. As parameter $A$ declines over time, infant mortality is reduced. If parameter $B$ remains at a low level, there would still be an important mortality decrease between ages zero and one. However, it is more reasonable to assume that mortality rates at young ages are getting closer to each other, as medical progresses with regard to birth conditions are more important than other medical advances. It is then preferred to keep the parameter $B$ constant at its median level (Fig. 2b).

Parameter $C$ : Its evolution is correlated with the one of the parameter $A$. Past medical progresses with regard to birth conditions were more important than other medical progresses. The infant mortality is then reduced more abruptly than the child mortality. Therefore, parameters $A$ and $C$ decrease. The decline in $A$ reduces the level of the infant and child mortality, while the decrease in $C$ brings the rates closer to each other. Thus, the gap in mortality rates between age zero and age ten is larger in the 1900s than in the 1970s. This decrease is modeled with an exponential function (Fig. 2c; Tables 1, 2).

Parameter $D$ : At the beginning of the twentieth century, medical progresses led to the decrease of the general level of mortality, the accident hump being affected as well. Once again, the exponential form is the most suitable one and is fitted over the period 1910-1979 (Fig. 2d; Tables 1, 2). An interesting remark is that the only aberrant value refers to 1918 , the year of the Spanish flu. It is the only 


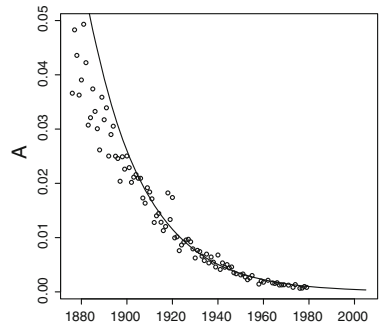

(a)

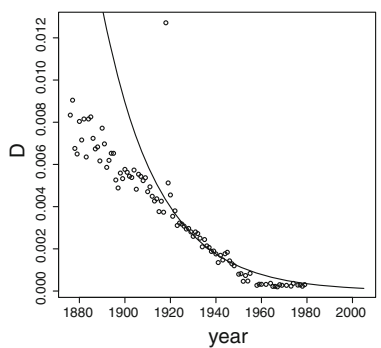

(d)

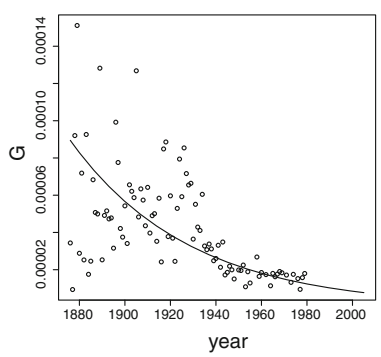

(g)

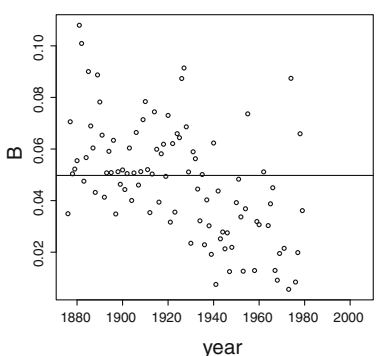

(b)

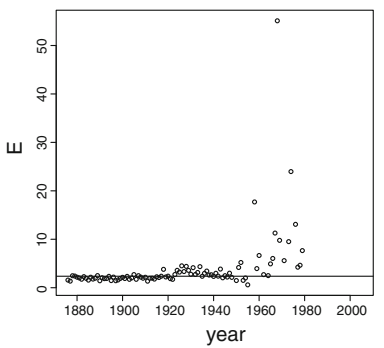

(e)

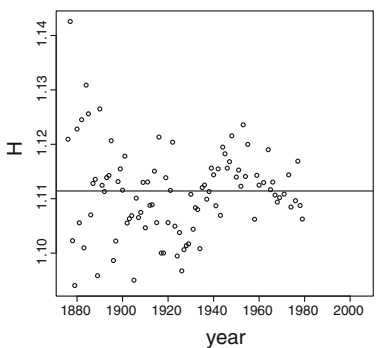

(h)

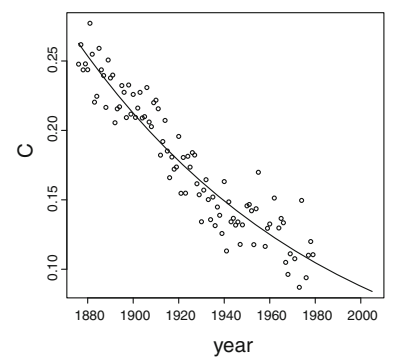

(c)

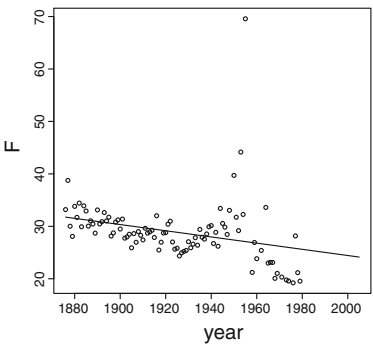

(f)

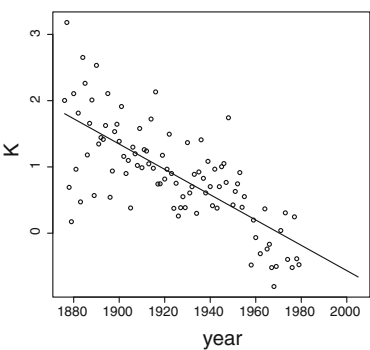

(i)

Fig. 2 Evolution of the Heligman-Pollard's parameters, females. The estimation of the HeligmanPollard function over the period 1876-1979 yields a set of observations on each parameter over time. Univariate models described in Sect. 4.1.2 are then applied to the fitted values of each parameter in order to forecast their temporal evolution until 2005 and thus to project the complete age profile of mortality. a Parameter $A$, b Parameter $B$, c Parameter $C$, d Parameter $D$, e Parameter $E$, f Parameter $F$, g Parameter $G, \mathbf{h}$ Parameter $H$, i Parameter $K$

parameter which is affected by it. It confirms the already known fact that this flu was deadly mainly among young adults.

Parameter $E$ : This evolution is more complicated to model. Indeed, since the 1960 s, the value increases. The quadratic and linear functions have significant coefficients at a one percent significance level. An increasing value for $E$ causes a concentration of the accident hump on a narrower band of ages, which implies a more visible hump. Notwithstanding, it seems more appropriate to assume a hump decreasing over time and becoming less noticeable. Under such considerations, the median value is preferred (Fig. 2e). 
Parameter $F$ : The coefficients of a quadratic function are non significant. The linear and exponential functions lead to similar results, that is a decreasing age at which the accident hump has the largest effect. From further analysis, the linear function is retained (Fig. 2f; Tables 1, 2).

Parameter $G$ : The most suitable function to fit this parameter appears to be the quadratic form (Table 2). However, an increasing value for $G$ would imply an unreasonably high level of mortality at older ages in future. Since the level of parameter $G$ becomes to some degree more stable over time, it looks more appropriate to fit an exponential function over the period 1935-1979 (Fig. 2g; Tables 1, 2). The decrease states that senior citizens live longer. Besides, the small number of older-age survivors at the beginning of the observed period explains the large variations in value.

Parameter $H$ : The coefficients of linear and exponential functions are clearly non significant, while the ones of a quadratic function are significant at a five percent significance level. However, a quadratic function would lead to a decreasing value for $H$ in future and so an impossibly low mortality level at older ages. Besides, this parameter oscillates around a constant level, the fluctuations being small in relative terms and consequently, the median value is applied in future years (Fig. 2h). Similarly to the parameter $G$, its value becomes more stable over time, and the important fluctuations we observe during the first years may be due to the small size of the population.

Parameter $K$ : As the coefficients of a quadratic function are non significant at a ten percent significance level, the most suitable model appears to be the linear one (Fig. 2i; Tables 1, 2). Parameter $K$ is positive until the 1960s, implying that the logarithm of the Heligman-Pollard function is concave downward at older ages. Then, $K$ gets negative and thus, the logarithm of mortality rates at older ages follows an exponential trend.

\subsubsection{Step 3: mortality forecasts}

The mortality rates are found with the Heligman-Pollard formula (Eq. 3), using the projected values of the parameters. In Fig. $3 \mathrm{a}-\mathrm{c}$, the circles define the observed mortality rates, while the curves represent the projections. As it can be noticed, the model overestimates a bit the mortality rates around age ten.

The accident hump seems to be a temporary element and, thereby, to affect the mortality rates only on a short term basis. It is already diminishing and will probably disappear in the coming years. In the future, it might be possible to remove the elements of Eq. 3 related to this hump.

\subsection{Lee-Carter model}

\subsubsection{Step 1: fit}

As previously mentioned, the model is fitted over the period 1950-1979 by maximum likelihood. Besides, the last ages are grouped under "100 and over", as 
Table 1 Estimates of the curves fitted to the parameters of the Heligmai-Pollard model, females

\begin{tabular}{|c|c|c|c|c|}
\hline Parameters & Estimate & Std error & $\mathrm{t}$ value & $\mathrm{p}$ value \\
\hline \multicolumn{5}{|c|}{ Parameter $A$ - exponential function } \\
\hline $\mathrm{a}$ & -0.04093 & 0.00206 & -19.86 & $<2 \mathrm{E}-16$ \\
\hline $\mathrm{b}$ & 0.07259 & 0.00709 & 10.24 & $8.67 \mathrm{E}-15$ \\
\hline \multicolumn{5}{|c|}{ Parameter $D$-exponential function } \\
\hline $\mathrm{a}$ & -0.04063 & 0.00502 & -8.092 & $3.41 \mathrm{E}-11$ \\
\hline $\mathrm{b}$ & 0.02471 & 0.00589 & 4.195 & $9.14 \mathrm{E}-05$ \\
\hline \multicolumn{5}{|c|}{ Parameter $G$-exponential function } \\
\hline $\mathrm{a}$ & -0.01903 & 0.00305 & -6.25 & $3.64 \mathrm{E}-07$ \\
\hline b & 0.00009 & 0.00002 & 4.355 & $1.11 \mathrm{E}-04$ \\
\hline \multicolumn{5}{|c|}{ Parameter $C$-exponential function } \\
\hline $\mathrm{a}$ & -0.00884 & 0.00033 & -26.71 & $<2 \mathrm{E}-16$ \\
\hline $\mathrm{b}$ & 0.26502 & 0.00389 & 68.21 & $<2 \mathrm{E}-16$ \\
\hline \multicolumn{5}{|c|}{ Parameter $F$-linear function } \\
\hline $\mathrm{a}$ & -0.05902 & 0.02003 & -2.95 & $4.05 \mathrm{E}-03$ \\
\hline b & 142.47743 & 38.55462 & 3.70 & $3.69 \mathrm{E}-04$ \\
\hline \multicolumn{5}{|c|}{ Parameter $K$-linear function } \\
\hline $\mathrm{a}$ & -0.01910 & 0.00182 & -10.47 & $<2 \mathrm{E}-16$ \\
\hline $\mathrm{b}$ & 37.63755 & 3.51132 & 10.72 & $<2 \mathrm{E}-16$ \\
\hline
\end{tabular}

All coefficients are significant at a one percent level

the model can not be applied at single older ages (missing data). The fitted model is introduced in Fig. 4, while the parameters resulting from the maximum likelihood estimation are presented in Fig. 5.

\subsubsection{Step 2: projection of $\kappa_{t}$}

Lee and Carter [12] suggest the use of a simple random walk with a drift, that is an $\operatorname{ARIMA}(0,1,0)$ process, in order to model the time series $\kappa_{t}$. Following the procedure described by Pandit and Wu [19], our analysis confirms an $\operatorname{ARIMA}(0,1,0)$ process with a drift of -2.15 as the best model (Fig. 5c).

\subsubsection{Step 3: mortality forecasts}

The mortality projections resulting from the Lee-Carter model are introduced in Fig. 3d-f. The main difference with the forecasts resulting from the HeligmanPollard (HP) model is reflected around the age of 20, since in the HP model, we assume a decreasing impact of the accident hump. 
Table 2 Bayesian information criterion of the curves fitted to the parameters of the HeligmanPollard model, females

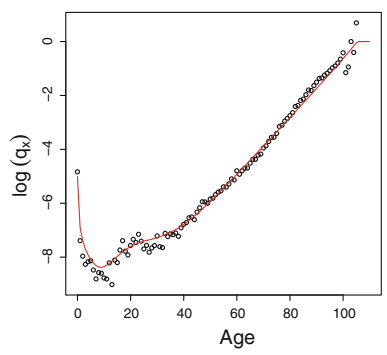

(a)

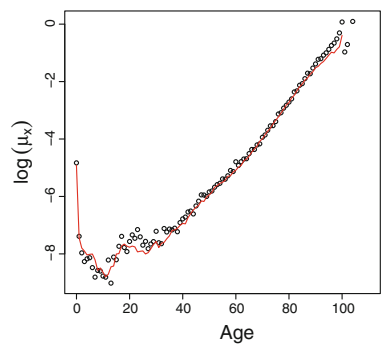

(d)

\begin{tabular}{lccc}
\hline Bayesian information criterion & & \\
\hline Parameters & Exponential & Linear & Quadratic \\
\hline A & -630 & -583 & -626 \\
C & -514 & -502 & -511 \\
D & -651 & -644 & -650 \\
F & 620 & 620 & 624 \\
G & -795 & -792 & -798 \\
K & 174 & 160 & 161 \\
\hline
\end{tabular}

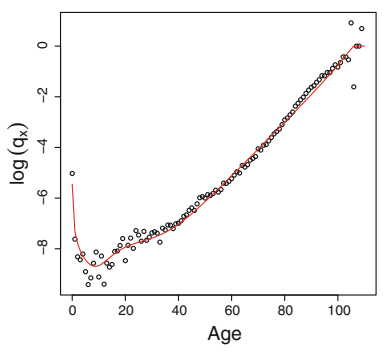

(b)

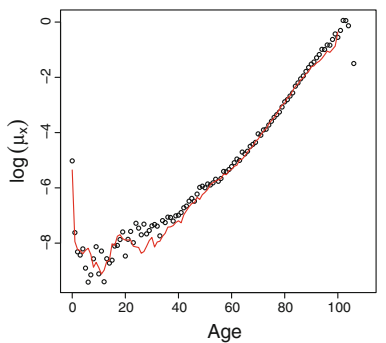

(e)

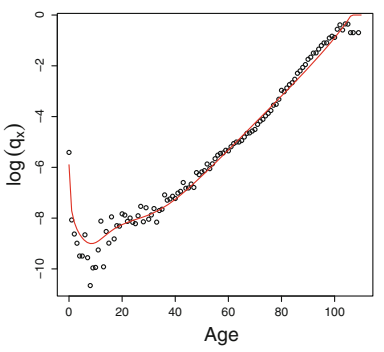

(c)

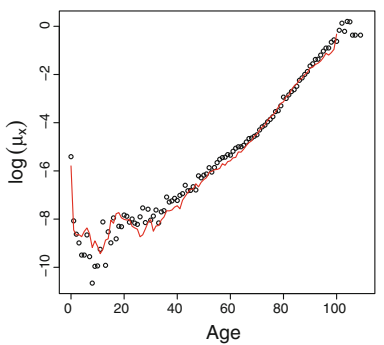

(f)

Fig. 3 Forecasted mortality rates over the period 1980-2005, females. Resulting forecasted mortality rates according to the Heligman-Pollard model (HP) or the Lee-Carter one (LC), as described in Sect. 4 circles reflect the observed mortality rates, while the curves are the projections. The last age-group for the Lee-Carter model was set at 100 and over, as the model could not be applied at older ages (missing data for older ages). a HP, 1980, b HP, 1990, c HP, 2000, d LC, 1980, e LC, 1990, f LC, 2000

\subsection{Model validations}

The forecasts are further compared with the actual data over the period 1980-2005. The life expectancies at birth and at age 65 are presented in Fig. 6. Circles define the observed life expectancies, while the curves reflect the forecasted ones. With both models, projections follow accurately the data.

Beside demographic statistics, pension funds are more interested in measuring the impact of the mortality forecasts on their retirement liabilities. Thus, the net single premium of a whole life annuity-due calculated using forecasted mortality is 


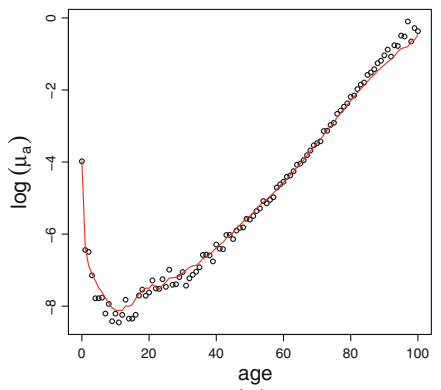

(a)

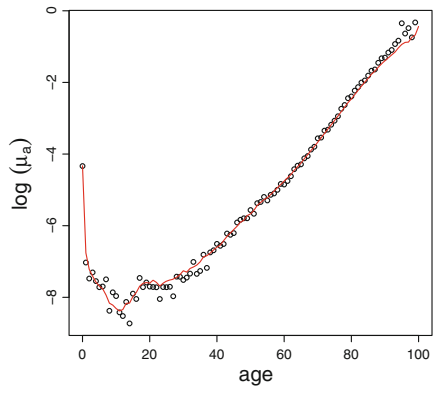

(b)

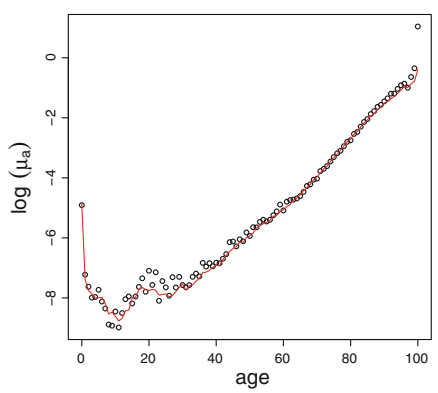

(c)

Fig. 4 Sample years of actual log-mortality rates (dots) and fitted Lee-Carter model (curve), females. a 1960 , b 1970 , c 1979

compared with the net single premium based on actual mortality. The net single premium of this annuity is defined as

$$
\ddot{a}_{x}=\sum_{k=0}^{\infty}(1+i)^{-k} \cdot{ }_{k} p_{x},
$$

where

$$
\begin{aligned}
{ }_{k} p_{x} & =\text { probability of surviving between ages } x \text { and } x+k ; \\
i & =\text { interest rate; } \\
& =0.04 .
\end{aligned}
$$

The results are presented in Table 3. The differences between the two models and the actual data are smaller than one percent. It confirms that these two models are good approximations of the reality in Switzerland, as already demonstrated in several studies for other countries (Tuljapurkar et al. [24], McNown and Rogers [14]).

For illustration purpose, the Lee-Carter model is also fitted over the entire period under observation, 1876-1979, and projections are performed using the new parameter values. As expected, fixing $\beta_{x}$ over a long fitting period imposes past 


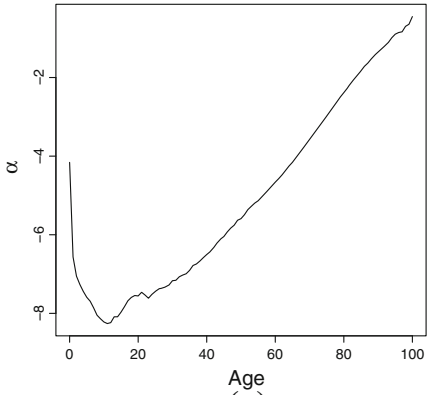

(a)

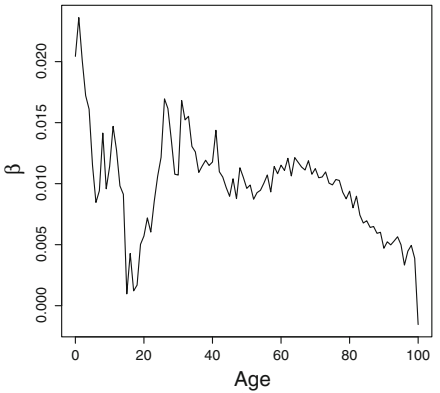

(b)

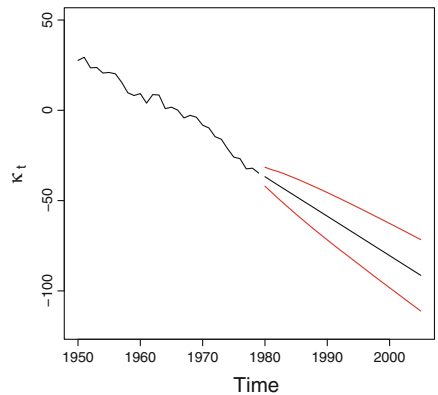

(c)

Fig. 5 Parameters of the Lee-Carter model, females. The Lee-Carter model is fitted to Swiss mortality rates over the period 1950-1979. The resulting parameters are introduced in this figure. Parameter $\kappa_{t}$ is forecasted until 2005 using the $\operatorname{ARIMA}(0,1,0)$ process with a drift of -2.15 . A confidence interval of 95\% for the projection is presented as well in the graph. a Parameter $\alpha_{x}$, b Parameter $\beta_{x}$, $\mathbf{c}$ Parameter $\kappa_{t}$

trends from long ago into the future which is clearly not valid. Indeed, the relative speed of decline at different ages may vary through time (Lee and Miller [13]). It highlights the need for care in how a forecasting method is applied.

\section{Future trends}

The analysis performed in previous sections suggests that the Heligman-Pollad and Lee-Carter models are both reliable to model Swiss mortality. We may then wonder what future trends in regard to mortality these models will forecast. In this section, the univariate models applied to the parameters of the Heligman-Pollard function and Lee-Carter model are re-estimated through 2005 to produce forecasts of mortality through 2075. Male and female projections are performed independently. Demographic results are presented in this section, while impacts on pension liabilities are introduced in the following one. Only representative forecasts are discussed in the analysis in order to highlight the key results. Graphs and tables for all statistics and forecasted years are available from the author upon request. 


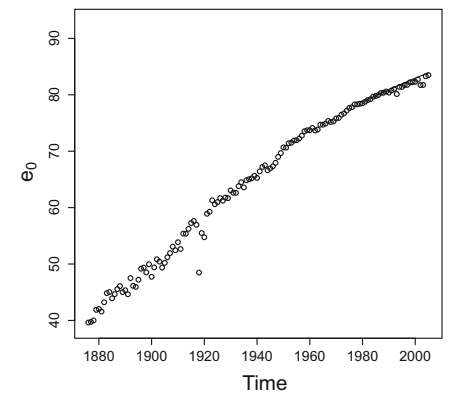

(a)

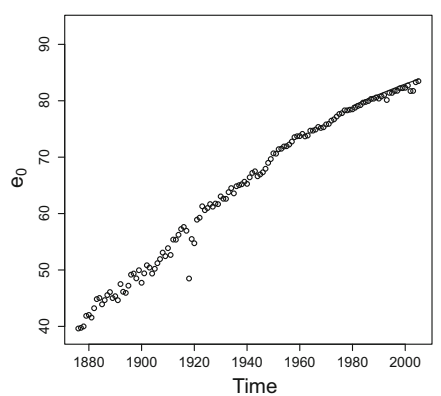

(c)

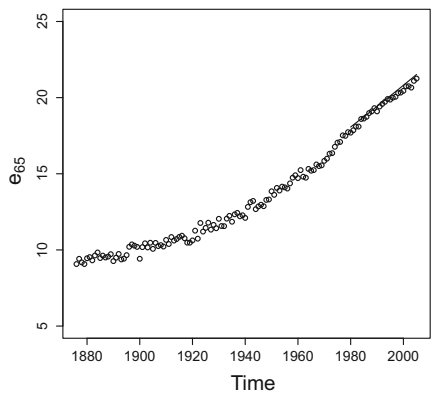

(b)

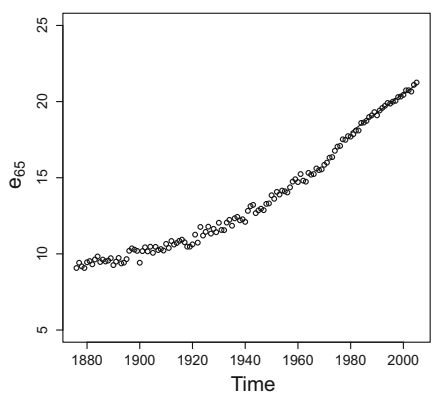

(d)

Fig. 6 Forecasted life expectancy over the period 1980-2005, females. Resulting forecasted life expectancy according to the Heligman-Pollard model (HP) or the Lee-Carter one (LC), as described in Sect. 4. Circles reflect the observed life expectancy from 1876 to 2005, while the curves are the projections. a Forecasted life expectancy at birth, HP. b Forecasted life expectancy at age 65, HP. c Forecasted life expectancy at birth, LC. d Forecasted life expectancy at age 65, LC

\subsection{Model fit}

\subsubsection{Heligman-Pollard}

Re-estimation of the univariate models applied to the parameters of the HeligmanPollard function for females yields only small differences from the results reported in Sect. 4.1.2, except for parameters $B, C$ and $E$. Indeed, the three parameters start to increase after the 1980s. The exponential function appears then to be the most appropriate one to model parameters $B$ and $E$, while the quadratic function is used for parameter $C$.

Male mortality differs substantially from female mortality especially at middle and older ages, which is reflected in the time series of the parameters of the Heligman-Pollard function. First, the age pattern of mortality displays a steeper curve at older ages, but at a lower level for females than for males (parameter $H$ is higher and $G$ lower for females). Parameter $K$ for males does not follow a clear structure, fluctuating mostly between minus one and two, and thus, Eq. 1 is fitted, that is without including parameter $K$ in the model. Second, the accident hump affects more male mortality, parameter $D$ being higher. From the 1980s, the accident hump is shifted to older ages (parameter $F$ increases and takes a value close 
Table 3 Net single premium of a whole life annuity-due at age 65 , females

\begin{tabular}{|c|c|c|c|c|c|c|c|}
\hline Year & $\begin{array}{l}\text { Real } \\
(\mathrm{X})\end{array}$ & $\begin{array}{l}\text { Computed } \\
\text { HP (Y) }\end{array}$ & $\begin{array}{l}\text { Delta }(\mathrm{HP}) \\
=(\mathrm{X}-\mathrm{Y}) / \\
\mathrm{X}(\%)\end{array}$ & $\begin{array}{l}\text { Computed } \\
\text { LC (Z), } \\
1876-1979\end{array}$ & $\begin{array}{l}\text { Delta (LC) } \\
=(\mathrm{X}-\mathrm{Z}) / \\
\mathrm{X}(\%)\end{array}$ & $\begin{array}{l}\text { Computed } \\
\text { LC (W) } \\
1950-1979\end{array}$ & $\begin{array}{l}\text { Delta }(\mathrm{LC}) \\
=(\mathrm{X}-\mathrm{W}) / \\
\mathrm{X}(\%)\end{array}$ \\
\hline 1980 & 12.69 & 12.78 & -0.75 & 12.56 & 0.97 & 12.81 & -0.94 \\
\hline 1981 & 12.78 & 12.85 & -0.53 & 12.60 & 1.38 & 12.87 & -0.72 \\
\hline 1982 & 12.90 & 12.91 & -0.09 & 12.64 & 2.01 & 12.94 & -0.29 \\
\hline 1983 & 12.90 & 12.97 & -0.60 & 12.68 & 1.71 & 13.00 & -0.81 \\
\hline 1984 & 13.13 & 13.04 & 0.72 & 12.71 & 3.19 & 13.07 & 0.51 \\
\hline 1985 & 13.13 & 13.10 & 0.22 & 12.75 & 2.89 & 13.13 & 0.01 \\
\hline 1986 & 13.20 & 13.16 & 0.27 & 12.79 & 3.12 & 13.19 & 0.06 \\
\hline 1987 & 13.30 & 13.23 & 0.54 & 12.82 & 3.57 & 13.25 & 0.34 \\
\hline 1988 & 13.36 & 13.29 & 0.49 & 12.86 & 3.71 & 13.32 & 0.30 \\
\hline 1989 & 13.47 & 13.35 & 0.84 & 12.90 & 4.24 & 13.38 & 0.66 \\
\hline 1990 & 13.37 & 13.42 & -0.35 & 12.93 & 3.27 & 13.44 & -0.52 \\
\hline 1991 & 13.51 & 13.48 & 0.23 & 12.97 & 4.02 & 13.50 & 0.08 \\
\hline 1992 & 13.59 & 13.54 & 0.35 & 13.00 & 4.32 & 13.56 & 0.22 \\
\hline 1993 & 13.66 & 13.61 & 0.38 & 13.04 & 4.53 & 13.62 & 0.27 \\
\hline 1994 & 13.74 & 13.67 & 0.48 & 13.08 & 4.80 & 13.68 & 0.40 \\
\hline 1995 & 13.72 & 13.73 & -0.05 & 13.11 & 4.47 & 13.74 & -0.10 \\
\hline 1996 & 13.80 & 13.79 & 0.01 & 13.15 & 4.71 & 13.80 & 0.02 \\
\hline 1997 & 13.82 & 13.86 & -0.28 & 13.18 & 4.61 & 13.86 & -0.27 \\
\hline 1998 & 13.94 & 13.92 & 0.13 & 13.22 & 5.18 & 13.91 & 0.18 \\
\hline 1999 & 13.95 & 13.98 & -0.20 & 13.25 & 5.04 & 13.97 & -0.13 \\
\hline 2000 & 14.00 & 14.04 & -0.29 & 13.28 & 5.13 & 14.03 & -0.18 \\
\hline 2001 & 14.13 & 14.10 & 0.20 & 13.32 & 5.77 & 14.08 & 0.35 \\
\hline 2002 & 14.14 & 14.17 & -0.17 & 13.35 & 5.59 & 14.14 & 0.03 \\
\hline 2003 & 14.10 & 14.23 & -0.87 & 13.39 & 5.10 & 14.19 & -0.64 \\
\hline 2004 & 14.29 & 14.29 & 0.00 & 13.42 & 6.09 & 14.25 & 0.28 \\
\hline 2005 & 14.36 & 14.35 & 0.10 & 13.45 & 6.35 & 14.30 & 0.43 \\
\hline
\end{tabular}

Real represents a whole life annuity-due at age 65 computed with actual mortality rates, Computed HP represents a whole life annuity-due at age 65 computed with forecasted mortality rates under the HeligmanPollard model, Delta $(H P)$ represents a gap in a whole life annuity-due at age 65 between the actual data and the Heligman-Pollard model, Computed $L C$ represents a whole life annuity-due at age 65 computed with forecasted mortality rates under the Lee-Carter model, Delta $(L C)$ represents a gap in a whole life annuitydue at age 65 between the actual data and the Lee-Carter model. The last ages are grouped under " 100 and over" for the Lee-Carter model, as this model can not be applied at older ages (missing data)

to 30 in 2000, while it was close to 20 in the 1980s), which is expected to reflect Human Immunodeficiency Virus (HIV) at the beginning of the 1980s, as this virus was mostly diagnosed on young males. Parameter $F$ is modeled with a quadratic function. The impact of the accident hump on males is also noticed with parameter $E$. From the 1900 s, its value increases considerably until the 1980 s, followed by a decrease. The increase appears then to be unusual and temporary, and causes a concentration of the hump on a narrower age range. It could be due to the growth of car accidents arising from the development of these means of transportation. As 
from the 1980s the value decreases, one can expect it to reach again its original level in 2040 (assumed to be equal to the median over the period 1876-1899).

\subsubsection{Lee-Carter}

The Lee-Carter model is fitted as well over the period 1950-2005. The same procedure as in Sect. 4.2 is applied. The time trend $\kappa_{t}$ is approximated with an $\operatorname{ARIMA}(2,1,1)$ process for females and an $\operatorname{ARIMA}(1,1,0)$ for males, as summarized in Table 4.

\subsubsection{LPP 2010}

As previously mentioned, the new official mortality tables (LPP 2010) were released in Switzerland at the end of 2010. These mortality tables are based on the actual mortality of 14 large pension funds observed over the period 2005-2009. These mortality rates are assumed to reflect the mortality as of first of July 2007. For the following years, a forecasting option based on reduction factors is included in the tables. The reduction factors are issued from two works published by the statistical office of the canton Vaud (Menthonnex [16, 17]). In these works, Swiss mortality is forecasted until 2150, mainly based on expert knowledge. The percentage decrease (reduction factor) between mortality rates of two adjacent years issued from Menthonnex's work is then applied to the observed pension fund mortality of 2007 and thus mortality is forecasted until 2150 . We applied the same reduction factors to our dataset, so that mortality forecasts until 2075 can be performed.

\subsection{Results}

The resulting age profiles of mortality rates are introduced in Fig. 7 for the three models. As mortality for ages above 100 are non reliable for a population as small as in Switzerland, the models are fitted on ages up to 100. However, one of the features of a parametric function such as Heligman-Pollard is that it allows to forecast mortality for older ages and thus forecasts for ages up to 110 can be performed under this model. It is important to note that the forecasts resulting from the LeeCarter model and from the reduction factors of Menthonnex's work are not smooth in Fig. 7. However, smoothing methods are applied in the LPP 2010 tables and

Table 4 Fitted ARIMA $(p, d, q)$ processes on parameter $\kappa_{t}$ of the Lee-Carter model

\begin{tabular}{|c|c|c|c|c|c|c|}
\hline & \multicolumn{3}{|c|}{ Females, $\kappa_{t}: \operatorname{ARIMA}(2,1,1)$} & \multicolumn{3}{|c|}{ Males, $\kappa_{t}: \operatorname{ARIMA}(1,1,0)$} \\
\hline & Values & CI $95 \%$ & & Value & CI 95\% & \\
\hline$\mu$ & -1.92 & -2.29 & -1.55 & -1.73 & -2.14 & -1.31 \\
\hline$\varphi_{1}$ & -1.24 & -1.47 & -1.01 & -0.31 & -0.57 & -0.06 \\
\hline$\phi_{2}$ & -0.56 & -0.79 & -0.32 & & & \\
\hline$\phi_{1}$ & 0.94 & 0.79 & 1.10 & & & \\
\hline$\sum \varepsilon_{t}^{2}$ & 217.31 & & & 229.76 & & \\
\hline
\end{tabular}




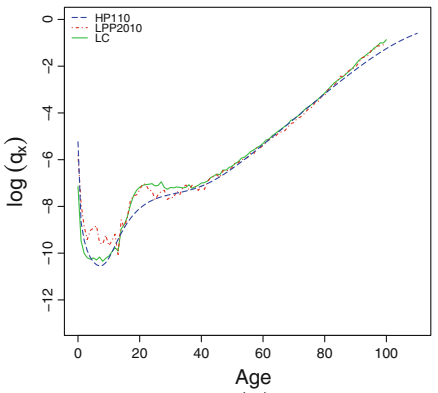

(a)

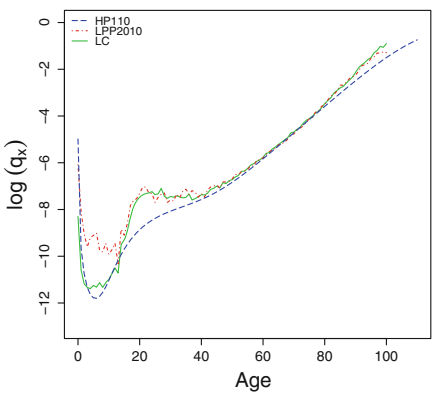

(c)

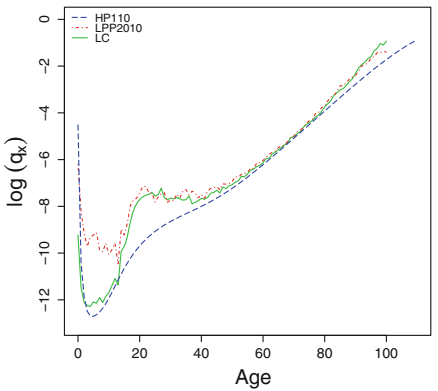

(e)

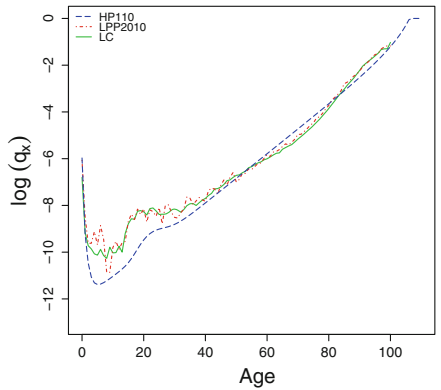

(b)

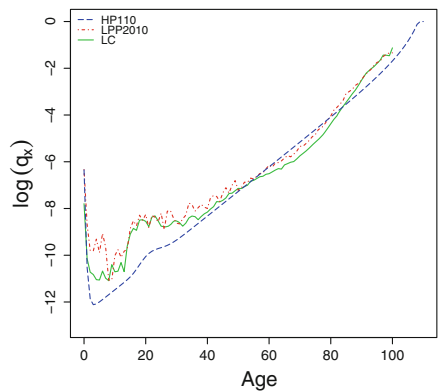

(d)

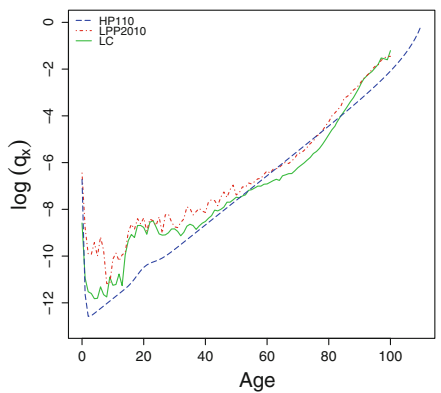

(f)

Fig. 7 Sample years of forecasted mortality. Resulting forecasted mortality according to the HeligmanPollard model (HP), the Lee-Carter model (LC) and the factors used in official tables used by pension funds (LPP 2010). The Heligman-Pollard model forecasts mortality for ages up to 110, while the other two models project mortality for ages up to 100. a 2030, males. b 2030, females. c 2055, males. d 2055, females. e 2075, males. f 2075, females

extensions of the Lee-Carter method may produce smooth forecasts as well. As these extended models have no impact on the analysis conducted thereafter, we choose to keep the simpler models previously introduced.

The assumption made in the Heligman-Pollard model of a decreasing impact of the accident hump is clearly reflected in Fig. 7. Indeed, mortality for ages 15-40 is forecasted to be lower with the Heligman-Pollard model. In contrast, the accident hump is emphasized in the Lee-Carter model. In the past 60 years, the accident 
hump appeared and so this trend is repeated in projections, as the Lee-Carter method assumes a constant pattern in the rate of change of mortality rates (represented by $\beta_{x}$ ). Besides, the Lee-Carter and Heligman-Pollard models forecast a more important decrease of mortality at young ages, especially for males. Even if the mortality for ages below 17 is not used by pension funds, it is interesting to note that Swiss experts from the statistical office expect a slow down in mortality decrease at young ages, which is not reflected in models using past evolutions to perform their forecasts. Finally, as already mentioned by Bongaarts [3], assuming a fixed relative speed of decline of mortality at different ages (a fixed $\beta_{x}$ over time) may be potentially problematic with the Lee-Carter model as differences in $\beta_{x}$ value for different ages may produce increasing differences in forecasted mortality across ages. This is particularly obvious with female forecasts for ages above 50 in Fig. 7. In contrast, with the Heligman-Pollard function, the age structure of mortality remains plausible, even with long time horizon forecasts.

A more convenient and intuitive way to compare these forecasts is through the life expectancy, introduced in Fig. 8. To make such a comparison, it is important to compute the life expectancies on the same basis for the three methods. Thus, the last age is set at 101 and is associated with a mortality rate of one. It is interesting to note the following: First, the Heligman-Pollard model forecasts the highest life expectancy at birth and at age 65 in the long-run. Second, the LPP 2010 tables assume a slow down in mortality improvements, as reflected in the life expectancy increasing at a slowing rate. This assumption is such that even if the forecasted life expectancy is higher for this model than the other two models at the beginning of the forecasting period (males), the Heligman-Pollard model projects a higher life expectancy at age 65 from 2022, while the Lee-Carter model is higher from 2066. Third, the differences between the two forecasting approaches presented in this paper and the one used in the LPP 2010 tables are more important for females. Even if it is not clear which projection is preferable, both models based on past evolutions predict further mortality decrease than the LPP 2010 tables.

In Fig. 8, life expectancy results from an age pattern with maximal age of 101 . Thus, this life expectancy does not truly reflect the complete mortality pattern as it is indeed possible to live until age 110 or even at older ages. As a parametric function allows to have mortality rates at older ages, we computed life expectancies including all ages until 110 and thus assuming that the probability to die at age 111 is one. The resulting 2075 life expectancies are introduced in Table 5. Including older ages increases life expectancy considerably. For ages below 100, a difference between half a year and one year and a half is noticed between the forecasted life expectancies of the two Heligman-Pollard models. At age 100, a more important gap is noticed, as one may expect. This table highlights how important are the assumptions used to forecast mortality for the centenarians.

\section{Impacts on future pension liabilities}

For a pension fund, it is even of more interest to measure the impact of the mortality assumptions on the valuation of pension liabilities. In Switzerland, the second pillar 


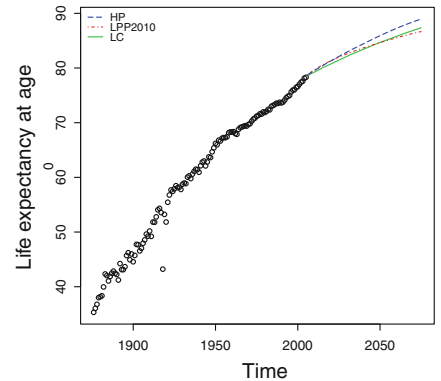

(a)

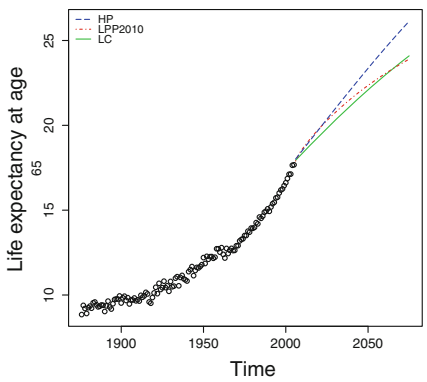

(c)

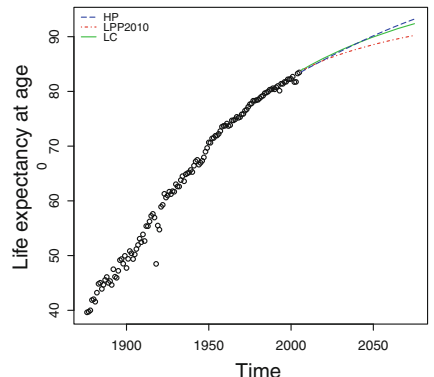

(b)

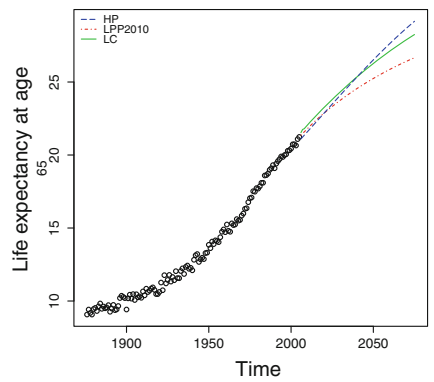

(d)

Fig. 8 Forecasted life expectancy. Resulting forecasted life expectancy according to the HeligmanPollard model (HP), the Lee-Carter model (LC) and the factors used in official tables used by pension funds (LPP 2010). We assume a probability of one to die at age 101. a Life expectancy at birth, males, b Life expectancy at birth, females, c Life expectancy at age 65, males, d Life expectancy at age 65, females

Table 5 Forecasted life expectancies, 2075

\begin{tabular}{|c|c|c|c|c|c|c|c|c|}
\hline \multirow[t]{2}{*}{ Age } & \multicolumn{4}{|l|}{ Males } & \multicolumn{4}{|l|}{ Females } \\
\hline & $\begin{array}{l}\text { Heligman- } \\
\text { polland, } \\
\text { age } 0-110\end{array}$ & $\begin{array}{l}\text { Heligman- } \\
\text { polland, } \\
\text { age } 0-100\end{array}$ & $\begin{array}{l}\text { Lee- } \\
\text { Carter, } \\
\text { age } \\
0-100\end{array}$ & $\begin{array}{l}\text { Lpp } \\
2010, \\
\text { age } \\
0-100\end{array}$ & $\begin{array}{l}\text { Heligman- } \\
\text { polland, } \\
\text { age } 0-110\end{array}$ & $\begin{array}{l}\text { Heligman- } \\
\text { polland, } \\
\text { age } 0-100\end{array}$ & $\begin{array}{l}\text { Lee- } \\
\text { Carter, } \\
\text { age } \\
0-100\end{array}$ & $\begin{array}{l}\text { Lpp } \\
2010, \\
\text { age } \\
0-100\end{array}$ \\
\hline 0 & 89.48 & 88.95 & 87.36 & 86.67 & 94.46 & 93.22 & 92.37 & 90.22 \\
\hline 10 & 80.47 & 79.94 & 77.37 & 76.92 & 84.58 & 83.34 & 82.40 & 80.42 \\
\hline 20 & 70.49 & 69.95 & 67.45 & 67.05 & 74.59 & 73.35 & 72.45 & 70.50 \\
\hline 30 & 60.56 & 60.03 & 57.78 & 57.41 & 64.61 & 63.37 & 62.55 & 60.63 \\
\hline 40 & 50.70 & 50.16 & 48.02 & 47.70 & 54.67 & 53.43 & 52.64 & 50.78 \\
\hline 50 & 40.91 & 40.37 & 38.28 & 38.01 & 44.81 & 43.57 & 42.80 & 41.03 \\
\hline 60 & 31.33 & 30.78 & 28.73 & 28.50 & 35.14 & 33.88 & 33.08 & 31.40 \\
\hline 70 & 22.23 & 21.66 & 19.62 & 19.44 & 25.85 & 24.56 & 23.45 & 21.97 \\
\hline 80 & 14.16 & 13.52 & 11.38 & 11.34 & 17.28 & 15.90 & 14.10 & 13.06 \\
\hline 90 & 7.83 & 6.92 & 4.94 & 5.38 & 9.93 & 8.24 & 6.20 & 6.09 \\
\hline 100 & 3.72 & 0.91 & 0.79 & 0.87 & 4.29 & 0.94 & 0.84 & 0.88 \\
\hline
\end{tabular}


law sets some minimal rules, and thus minimal pension amounts, that have to be followed by all pension funds. In this system, employees, and their employer, have to pay contributions to a fund. These contributions are accumulated in a saving account until the retirement age. At the retirement age, a factor, called the pension conversion rate, is applied to the accumulated capital in order to transform this capital into an annual pension amount. This conversion rate is now set at a level of $6.8 \%$. With the increase of life expectancy, many discussions hold among politicians, economists and actuaries in order to decide if this rate should be reduced. Thus, an interesting approach would be to determine the impact of the mortality assumptions on this pension conversion rate.

The retirement age is fixed at 65 for males and 64 for females in Switzerland. Thus, the pension conversion rate is defined as the inverse of the sum of four discounted annuities: first a discounted annuity value for a male age 65 (female age 64 ), second a $60 \%$ reversionary widow's (widower's) pension, third a $20 \%$ reversionary orphan's pension and forth a $20 \%$ pension for the children of a retired person. The discount rate for the annuities is set at $3.5 \%$ and children receive a pension until age 25 . The average number of children a person may have, the average age of the children a person may have, the probability to be married and the average age of the spouse are assumed constant over time and found in the EVK 2000 mortality tables, as the LPP 2010 tables were not made available to us. Besides, the mortality rates of a widow or widower differing from the mortality of the general population, the percentage differences between the general mortality and the widow/widower's mortality of the EVK 2000 tables are assumed constant over time and applied to the forecasted mortality introduced in previous sections.

Results are presented in Fig. 9. Two different conversion rates are introduced. In the upper graphs, conversion rates according to the mortality of a specified year are presented. In the two lower graphs, conversion rates of a specified generation are shown. For example, for the generation born in 1920, a male reaches the retirement age of 65 in 1985. The pension conversion rate for the 1920 generation is then based on the probability to die at age 65 in 1985, the probability to die at age 66 in 1986 and so on. If the age pattern of mortality includes ages up to 100, one needs 35 years of observation (or forecast) to compute the pension conversion rate, as there are 35 years from age 65 to age 100. Thus, the last generation for which the pension conversion rate is found is the generation reaching the retirement age of 65 in 2040, and thus born in 1975. If the age pattern of mortality includes ages up to 110 , one needs 45 years of observation (or forecast) so that the last generation for which we compute the pension conversion rate is the generation born in 1965. Past mortality is used until 2005, while the forecasted rates according to one of the three models are employed from 2006. For the generation born before the 1930s, the pension conversion rate is then mostly based on past mortality (observed mortality) which explains the fluctuations we observe for the first years in Fig. 9c, d.

According to the three models, a pension conversion rate of $6.8 \%$ is too high and will continue to decrease in the coming years. The two forecasting approaches based on past observations (Lee-Carter and Heligman-Pollard) forecast a more important decrease, especially for females. The more conservative assumptions lying in the 


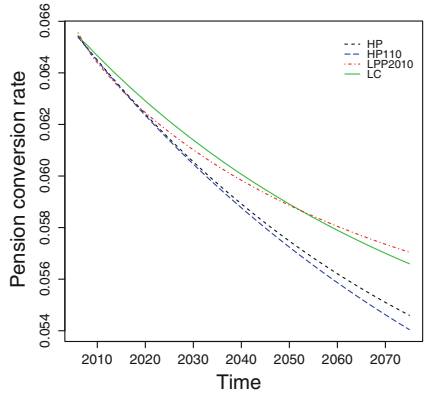

(a)

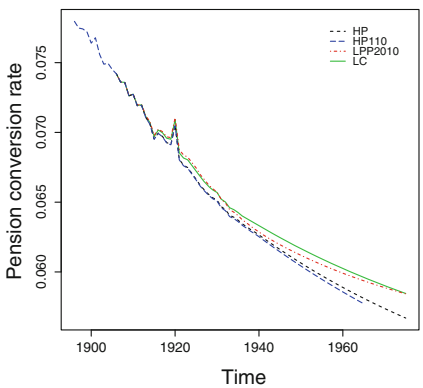

(c)

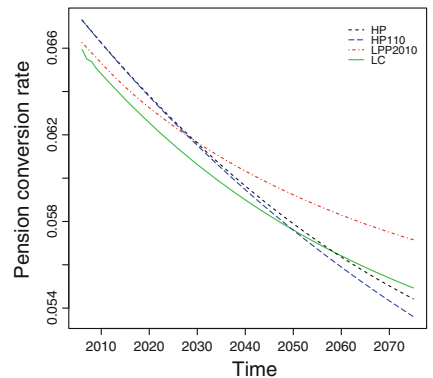

(b)

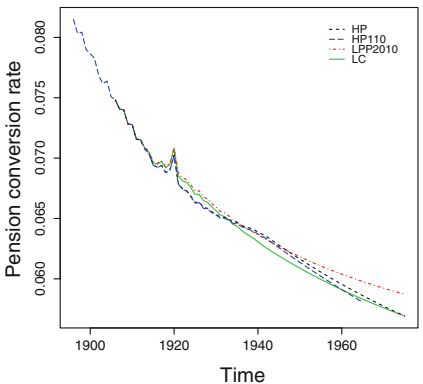

(d)

Fig. 9 Pension conversion rate according to the Swiss law. Resulting forecasted pension conversion rate according to the Heligman-Pollard model (HP), the Lee-Carter model (LC) and the reduction factors used in official tables used by pension funds (LPP 2010). We assume a probability of one to die at age 101, except for the curve "HP110" which takes into account mortality until age 110 and has a probability of one to die at age 111. Periodic table: the mortality rates of the year specified on the X-coordinate are used. Generation table: the X-coordinate indicates the year of birth. a Periodic table, males, age 65 , b Periodic table, females, age 64, c Generation table, males, age 65, d Generation table, females, age 64

forecasting approach applied in the LPP 2010 tables slow down the expected future mortality decline, which reflect the views of many experts across the world. However, past evolutions, if replicated in future, tend to indicate that mortality may decline more than expected.

Another important remark refers to the very old ages. When considering ages up to 110 in the Heligman-Pollard model, the forecasted decline of the pension conversion rate is emphasized over time. As mortality declines, a greater proportion of the population will reach the centenarian group. It will then become a more important matter to accurately model mortality for these ages.

We present a concrete example of the financial impact of the mortality assumptions for Swiss pension funds. A female reaches the retirement age with a saving account of CHF 500'000. According to the law, the pension fund should pay a retirement pension of CHF 34'000 per year $\left(500^{\prime} 000 \times 6.8 \%\right)$. If she reaches the retirement age in 2010, the periodic pension conversion rates according to the Heligman-Pollard model (last age set at 100 (HP100) or 110 (HP110)), the LeeCarter model (LC) or the LPP 2010 tables are 6.63, 6.62, 6.48 and 6.53\% respectively. The corresponding pensions are CHF 33'150, CHF 33'100, CHF 
32'400 and CHF 32'650. However, according to the generation table of a female born in 1946 and thus being age 64 in 2010, the pension should be as low as CHF 31'300 (pension conversion rate of 6.26\%, HP100), CHF 31'150 (6.23\%, HP110), CHF 30'850 (6.17\%, LC) and CHF 31'250 (6.25\%, LPP2010), that is an amount lower of around CHF 1'500-2'000 per year. If the retirement age of 64 is reached in 2029, the periodic tables suggest a pension of CHF 30'900 (6.18\%, HP100), CHF 30'850 (6.17\%, HP110), CHF 30'400 (6.08\%, LC) and CHF 30 '900 (6.18\%, LPP2010), while the generation tables would require a pension of CHF 29'300 (5.86\%, HP100), CHF 29'050 (5.81\%, HP110), CHF 29'150 (5.83\%, LC) and 29 '900 (5.98\%, LPP2010). It represents a decrease close to CHF 2'000 between the two periodic tables for the Heligman-Pollard and Lee-Carter models, while it is a decrease of only CHF 1'700 according to the LPP 2010 tables. The decreases are less important between the generation tables, namely around CHF 2'000, CHF 1'700 and CHF 1'300 for the Heligman-Pollard models, Lee-Carter model and LPP 2010 tables respectively. Finally, the periodic tables for 2075 give a pension of CHF 27’200 (5.44\%, HP100), CHF 26'800 (5.36\%, HP110), CHF 27'450 (5.49\%, LC) and 28'600 (5.72\%, LPP2010), which represent an annual decrease of around CHF 6'000 (HP100 and HP110), CHF 5'000 (LC) and 4'000 (LPP2010) compared to 2010 and even a further annual decline of CHF 1'000 if compared to the actual pension conversion rate of $6.8 \%$.

The above issue may be analyzed under a different angle. For a female to get a pension conversion rate of $6.8 \%$ in 2010 (that is a retirement pension of CHF 34'000 in previous example), the retirement age should be fixed at 65 according to the periodic-HP models or 66 for the other two periodic models. In accordance with the generation tables, a female retiring in 2010 should be between 67 and 68 . There are small differences between the three models at the beginning of the forecasting period. However, these differences increase over time, such that in 2075, the retirement age should be set at age 74 (HP100), 75 (HP110), 73 (LC) or 71 (LPP2010) for females. Due to the more important improvements in mortality under the HP and LC models, pension funds should then start to pay retirement pensions approximately three years later than what is expected with the LPP 2010 tables. For males, results are more similar: retirement age of 75 for both HP models and 73 for LC and LPP 2010 in 2075.

With this simple example, we want to highlight the need for alternative models. One can easily see that the current LPP 2010 mortality forecasts used give the most costly results. Among the models considered, the pension funds using LPP 2010 pay the highest amount per year or should start paying at a younger age, both leading to higher financial costs.

\section{Conclusion}

In this paper, we compare two mortality forecasting approaches developed in academia with the method currently applied in official mortality tables used by pension funds in Switzerland. Among the few approaches developed for mortality modeling, the Lee-Carter and Heligman-Pollard models are chosen for several 
reasons. First, they were successfully applied in the past in several countries (McNown and Rogers [14], Rogers and Gard [20], Tuljapurkar et al. [24]), and the tests conducted on Swiss data in Sect. 4 reveal good performances. Second, they can be easily fitted through maximum likelihood estimation (LC) or non-linear minimization (HP) using pre-existing functions developed in statistical programs such as $R$. Third, their structure is easily interpreted in demographic terms which eases the forecasting procedure. It allows to include specific assumptions about the future in the forecasting model. As an example, this study assumes a diminishing accident hump through the Heligman-Pollard model. Fourth, the proposed univariate models used to forecast the value of the parameters are more easily understood and applied than multivariate approaches.

In 2006, the statistical office of the canton Vaud already forecasted mortality based on data available until 2004 (Menthonnex [16]). In 2009, the office updated its forecasts using the data of 2005-2008 (Menthonnex [17]). By doing so, the expected future mortality improvements, and thus the life expectancy, increased. However, our results reveal that forthcoming mortality decrease may still be more important than these revised forecasts, used in the LPP 2010 tables, especially for females. Over the second half of the 20th century, the improvement in life expectancy has been a consequence of a decrease in mortality at older ages, caused by progresses in cardiovascular diseases and tumors (Münz and Wanner [18]). As a consequence, the Lee-Carter and Heligman-Pollard models repeat for future years the improvement in mortality rates at older ages. If past evolutions are believed to continue in the future, the conservative approach applied in the LPP 2010 tables may be inadequate, as the assumed slow down in the mortality decrease might be too consequent.

Financial consequences were also enlightened. Depending on the chosen forecasting approach, pension funds might have to pay higher pensions than what they can afford. It is then important to consider several forecasting models, including models developed in academia. Several alternatives should be considered when analyzing the impact of mortality risk on the financial stability of a pension fund.

Dynamic mortality rates are then important considerations for actuarial valuations. It increases the adequacy between the contributions paid by the employees and employers and the pensions paid, in return, by the pension funds. However, some cautions are required with the use of mortality forecasts, which results in the following suggestions.

First, the fitting period of a chosen model needs to be carefully selected. As discussed in Sect. 4.3 and demonstrated with the Lee-Carter model, using a long fitting period imposes past trends from long ago into the future, an assumption which may not hold over some extended periods. As a consequence, we suggest to fit a model over the complete time frame, but as well on smaller sets. The period 1950 onward gives good results, as in other countries (Lee and Miller [13]), and thus, should also be considered.

Second, convention and caution do not recommend to forecast mortality over a period longer than the fitting period (Booth et al. [4]). Indeed, a model can not reflect the whole historical and future profile of mortality. For example, in the LeeCarter model, the trend of $\kappa_{t}$ can not be valid over the complete historical period as 
it would lead to impossibly high mortality rates in the eighteenth century. The same analysis can be applied to the evolutions of the Heligman-Pollard parameters. Parameters $A, D$ and $G$ follow an exponential decrease and thus, tend to infinity as we go further in the past. It was then decided not to forecast mortality after 2075. Consequently, the forecasting mortality approach available in the LPP 2010 tables and allowing to project mortality until 2150 seems non-reliable. Even if generation tables are theoretically appealing for actuarial valuations used in pension funds, such as for the "best estimate" requirements of the IAS19 (International Accounting Standard No 19) ${ }^{4}$, they need mortality forecasts over a too extended time horizon to be fully reliable. If a pension fund still wishes to use generation tables, its shortcomings should be kept in mind and the forecasts regularly updated with new data.

Third, we recommend to forecast mortality with several models, as it gives a broader picture of the possible future. They may be looked as different scenarios. Another option would be to forecast probabilistic intervals along with the expected values. The Lee-Carter model allows to do it easily as the forecast of the parameter $\kappa_{t}$ contains a forecast interval issued from the univariate time series theory (as in Fig. 5c). However, several sources of errors exist (such as model misspecification and parameter estimation), some of them not being included in the model proposed by Lee and Carter [12]. Thus, their prediction interval is known to be overly narrow. The accuracy of forecast intervals has been widely studied recently for several models and an interesting review of this matter is found in Booth and Tickle [5]. Forecasting models based on essentially non-statistical methods, especially the use of "informed judgement" in projecting mortality, do not offer the nice feature of prediction intervals. Therefore, assessing the precision of the forecasts used in the LPP 2010 tables is more difficult. However, as previously mentioned, several scenarios may give a good estimation of such intervals.

Finally, our last suggestion refers to the use of a summary statistic such as the life expectancy. Depending on the last age included in the life tables, result may vary. This issue will become even more important in future as the probability for a person to live until age 100 and over is increasing. Thus, assumptions on future mortality rates for the centenarians are key components and have important impacts on life expectancy and discounted annuity values. As data for these ages are missing, only expert opinions or senescent mortality models (such as Coale and Kisker [6] or Bongaarts [3]) may be used. We recommend then to compute life expectancies and discounted annuity values first excluding and second including the very old ages. It gives the forecaster a good approximation of the impact assumptions on centenarians have on pension liabilities.

Acknowledgments The author acknowledges Corina Constantinescu for her precious and very useful comments on the paper.

\footnotetext{
4 The IAS19 requires to set assumptions on future mortality rates that should represent the "best estimates". If the life expectancy is assumed to increase in the coming years, generation tables are perceived as "best estimates".
} 


\section{References}

1. Bell WR (1997) Comparing and assessing time series methods for forecasting age-specific fertility and mortality rates. J Off Stat 13(3):279-303

2. The Department of Demography at the University of California, The Max Planck Institute for Demographic Research (2007) Human mortality database. http://www.mortality.org

3. Bongaarts J (2005) Long-range trends in adult mortality: models and projection methods. Demography 42(1):23-49

4. Booth H, Maindonald J, Smith L (2002) Applying Lee-Carter under conditions of variable mortality decline. Popul Stud 56(3):325-336

5. Booth H, Tickle L (2008) Mortality modelling and forecasting: a review of methods. Ann Actuar Sci 3:3-43

6. Coale A, Kisker E (1990) Defects in data on old age mortality in the United States: new procedures for calculating approximatively accurate mortality schedules and life tables at the highest ages. Asian Pac Popul Forum 4:1-31

7. Delwarde A, Denuit M (2006) Construction de Tables de Mortalité Périodiques et Prospectives. Economica, Paris

8. Felipe A, Guillen M, Perez-Marin AM (2002) Recent mortality trends in the Spanish population. Br Actuar J 8:757-786

9. Gaille S, Sherris M (2011) Modelling mortality with common stochastic long-run trends. Geneva Pap Risk Insur Issues Pract 36(4):595-621

10. Heligman L, Pollard JH (1980) The age pattern of mortality. J Inst Actuar 107:49-80

11. Lee RD (2000) The Lee-Carter method of forecasting mortality, with various extensions and applications. North Am Actuar J 4:80-93

12. Lee RD, Carter L (1992) Modeling and forecasting US mortality. J Am Stat Assoc 87:659-671

13. Lee RD, Miller T (2001) Evaluating the performance of the Lee-Carter approach to modeling and forecasting mortality. Demography 38:537-549

14. McNown R, Rogers A (1989) Forecasting mortality: a parameterized time series approach. Demography 26(4):645-660

15. McNown R, Rogers A (1992) Forecasting cause-specific mortality using time series methods. Int J Forecast 8:413-432

16. Menthonnex J (2006) Tables de mortalité longitudinales pour la Suisse. Technical Report, SCRIS

17. Menthonnex J (2009) La Mortalité par Génération en Suisse. Technical Report, SCRIS

18. Münz R, Wanner P (2006) Démographie: Ce qui Nous Attend Demain. Evolutions Globales, Tendances Européennes et Vieillissement en Suisse. Technical Report, Avenir Suisse

19. Pandit SM, Wu SM (2001) Time series and system analysis with applications. Krieger, Malabar

20. Rogers A, Gard K (1991) Applications of the Heligman/Pollard model mortality schedule. Popul Bull U N 30:79-105

21. Tabeau E, Ekamper P, Huisman C, Bosch A (1999) Improving overall mortality forecasts by analysing cause-of-death, period and cohort effects in trends. Eur J Popul 15(2):153-183

22. Tabeau E, Van Den Bergh Jeths A, Heathcote C (2001) Forecasting mortality in developed countries. Insights from a Statistical, Demographic and Epidemiological Perspective. Kluwer Academic Publishers, Dordrecht

23. Thiel PN (1872) On a mathematical formula to express the rate of mortality throughout the whole life. J Inst Actuar 16:313-329

24. Tuljapurkar S, Li N, Boe C (2000) A universal pattern of mortality decline in the G7 countries. Nature 405:789-792 